\title{
Oscillation of First-order Neutral Difference Equation
}

\author{
Xiaohui Gong (Corresponding author) \\ Department of Science, Yanshan University \\ 438 West of He Bei Avenue, Qinhuangdao 066004, China \\ E-mail: gongxiaohui318@yahoo.com.cn \\ Xiaozhu Zhong, Jianqiang Jia, Rui Ouyang \& Hongqiang Han \\ Department of Science, Yanshan University \\ 438 West of He Bei Avenue, Qinhuangdao 066004, China
}

\begin{abstract}
The oscillation of the first order neutral difference equation

$\Delta[x(n)-p x(n-\tau)]+q x(n-\sigma)=0$

is studied in this paper,where $p \in(0,1)$ and $q, \tau, \sigma$ are positive constants. The sufficient conditions for oscillation of the equation are obtained by suitable inequality and characteristic equation.
\end{abstract}

Keywords: Difference equation, Neutral, Oscillation

\section{Introduction}

Qualitative behavior of solutions of difference equations has received considerable interest recently. In [1] the oscillation of the first order nertral difference equation

$\Delta[x(n)-p x(n-\tau)]+q x(n-\sigma)=0$,

was considered and some oscillation criteria were given, where $\Delta$ is the forward difference, i.e., $\Delta x_{n}=x_{n+1}-x_{n}, p \in(0,1)$, and $q, \tau, \sigma$ are positive constants. In this paper we assume $\tau-\sigma>1$ and $\tau-\sigma=1$ and study the oscillation for (1) in both cases and obtain further oscillatory criteria.

A solution of equation (1) is called oscillatory, if it is neither finally positive nor negative. Otherwise it is called nonoscillatory.

\section{2. main result}

Lemma $1^{[2]}$ A necessary and sufficient condition for all solutions of equation (1) to oscillate is that the characteristic equation

$F(\lambda)=(\lambda-1) \lambda^{\sigma-\tau}\left(\lambda^{\tau}-p\right)+q=0$

has no positive real root.

Theorem 1 Assume that $0<p<1$ and $\tau-\sigma>1$, a sufficient condition for every solution of equation (1) to oscillate is that $q-\frac{\tau}{\tau-\sigma-1}\left(1-p^{\frac{1}{\tau}}\right)>0$.

Proof: Since $F(\lambda)=(\lambda-1) \lambda^{\sigma-\tau}\left(\lambda^{\tau}-p\right)+q=0$ and $q>0$, the equation (2) possibly has roots only when $(\lambda-1) \lambda^{\sigma-\tau}\left(\lambda^{\tau}-p\right)<0$.

But $0<p<1$, when $\lambda \geq 1$ and $\lambda<p^{\frac{1}{\tau}}<1,(\lambda-1) \lambda^{\sigma-\tau}\left(\lambda^{\tau}-p\right)>0$ and the equation (2) has no positive 
solution. Therefore the equation (2) possibly has roots only on $\left(p^{\frac{1}{\tau}}, 1\right)$.

Since $F(\lambda)=(\lambda-1) \lambda^{\sigma-\tau}\left(\lambda^{\tau}-p\right)+q$ continuous when $\lambda \in\left[p^{\frac{1}{\tau}}, 1\right], F(\lambda)$ has greatest value and smallest value on interval $\left[p^{\frac{1}{\tau}}, 1\right]$.

Obviously, $F(1)=F\left(p^{\frac{1}{\tau}}\right)=q$ is greatest value, then there is at least a point $\xi \in\left(p^{\frac{1}{\tau}}, 1\right)$ such that $F(\xi)$ is a smallest value.

Evidently function $F(\lambda)=(\lambda-1) \frac{1}{\lambda^{\tau-\sigma}}\left(\lambda^{\tau}-p\right)+q=0,(\tau-\sigma>1)$ is continuous and derivables on interval $\left(p^{\frac{1}{\tau}}, 1\right)$, then $\xi$ is a minimum point also.

By Fermat's theorem we know $F^{\prime}(\xi)=0$, i.e.:

$$
F^{\prime}(\xi)=\xi^{\sigma-\tau}\left(\xi^{\tau}-p\right)+(\sigma-\tau)(\xi-1) \xi^{\sigma-\tau-1}\left(\xi^{\tau}-p\right)+(\xi-1) \xi^{\sigma-\tau} \tau \xi^{\tau-1}=0
$$

Obviously,

$$
\begin{aligned}
& \xi^{\tau}-p=-\frac{\tau(\xi-1) \xi^{\tau}}{\xi+(\sigma-\tau)(\xi-1)} \\
& F(\xi)=(\xi-1) \xi^{\sigma-\tau}\left(\xi^{\tau}-p\right)+q=(\xi-1) \xi^{\sigma-\tau}\left[-\frac{\tau(\xi-1) \xi^{\tau}}{\xi+(\sigma-\tau)(\xi-1)}\right]+q=q-\frac{\tau(\xi-1)^{2} \xi^{\sigma}}{\xi+(\sigma-\tau)(\xi-1)} .
\end{aligned}
$$

Let $\xi=1-s,(0<s<1)$,

$$
F(\xi)=q-\frac{\tau s^{2}(1-s)^{\sigma}}{1-s-(\sigma-\tau) s}=q-\frac{\tau s^{2}(1-s)^{\sigma}}{1+(\tau-\sigma-1) s}
$$

From $\tau-\sigma>1$ we have $\tau-\sigma-1>0$, then

$$
F(\xi)>q-\frac{\tau s^{2}(1-s)^{\sigma}}{(\tau-\sigma-1) s}=q-\frac{\tau s(1-s)^{\sigma}}{\tau-\sigma-1}=q-\frac{\tau(1-\xi) \xi^{\sigma}}{\tau-\sigma-1}>q-\frac{\tau\left(1-p^{\frac{1}{\tau}}\right)}{\tau-\sigma-1}>0
$$

Because $\xi$ is a smallest point, the equation(2) has no real root on interval $\left(p^{\frac{1}{\tau}}, 1\right)$. Therefore all solutions of equation (1) are oscillatory.

Theorem 2 Assume that $0<p<1, \tau-\sigma=1$ and $\tau>\frac{1}{2}$, a sufficient condition for every solution of equation (1) to oscillate is that $\sqrt{p q(2 \tau-1)}\left(\frac{2 \tau}{2 \tau-1}\right)^{\tau}>\frac{1}{2}$.

Proof: Obviously, the equation (2) has no real root when $\lambda \geq 1$, so if $F(\lambda)=0$ has real roots, it must be on $(0,1)$.

Let $u=\frac{1}{\lambda}$, then $h(u)=\frac{\mathrm{F}\left(\frac{1}{u}\right) u^{\tau}}{u-1}=p u^{\tau}-1+\frac{q u^{\tau}}{u-1} \geq 2 u^{\tau} \sqrt{\frac{p q}{u-1}}-1$.

We will prove that $h(u)$ has no zero point:

Let $h_{1}(u)=2 u^{\tau} \sqrt{\frac{p q}{u-1}}-1$, then $h_{1}(u)$ has minimum value $2 \sqrt{p q(2 \tau-1)}\left(\frac{2 \tau}{2 \tau-1}\right)^{\tau}-1$ at $u=1+\frac{1}{2 \tau-1}$.

However $2 \sqrt{p q(2 \tau-1)}\left(\frac{2 \tau}{2 \tau-1}\right)^{\tau}-1>0$, so $h(u)$ has no zero point and the equation(2) has no real root on interval $(0,1)$. Therefore all solutions of equation (1) are oscillatory.

Theorem 3 Assume that $0<p<1$ and $\tau-\sigma=1$, a sufficient condition for every solution of equation (1) to oscillate is that $q-\tau\left(p^{\frac{1}{\tau}}-1\right)^{2}>0$. 
Proof: We know that there is at least a point $\xi \in\left(p^{\frac{1}{\tau}}, 1\right)$, by theorem 1 ,such that $F(\xi)$ is a minimum value and $F^{\prime}(\xi)=0$, then $F(\lambda)=q-\frac{\tau(\xi-1)^{2} \xi^{\sigma}}{\xi+(\sigma-\tau)(\xi-1)}$

From $\tau-\sigma=1$ we have $F(\lambda)=q-\tau(\xi-1)^{2} \xi^{\sigma}$,

Since $\xi \in\left(p^{\frac{1}{\tau}}, 1\right), F(\lambda)=q-\tau(\xi-1)^{2} \xi^{\sigma}>q-\tau\left(p^{\frac{1}{\tau}}-1\right)^{2}>0$.

Because $\xi$ is a smallest point, the equation (2) has no real root on interval $\left(p^{\frac{1}{\tau}}, 1\right)$. Therefore all solutions of equation (1) are oscillatory.

\section{Examples}

Example 1.Consider difference equation $\Delta\left(x(n)-\frac{1}{4} x(n-2)\right)+3 x\left(n-\frac{1}{2}\right)=0$ where $p=\frac{1}{4}, \tau=2$,

$\sigma=\frac{1}{2}, q=3$. Then $\tau-\sigma=\frac{3}{2}>1, q-\frac{\tau}{\tau-\sigma-1}\left(1-p^{\frac{1}{\tau}}\right)=1>0$

so the conditions in theorem 1 are satisfied and the characteristic equation is

$$
F(\lambda)=(\lambda-1) \lambda^{-\frac{3}{2}}\left(\lambda^{2}-\frac{1}{4}\right)+3=0
$$

From the figure 1 we can see the equation (2) has no real root. Therefore all solutions of equation (1) are oscillatory.

Example 2.Consider difference equation $\Delta\left(x(n)-\frac{1}{10} x(n-2)\right)+10 x(n-1)=0$ where $p=\frac{1}{10}, \tau=2$,

$\sigma=1, q=10$. Then $\tau-\sigma=1$ and $\sqrt{p q(2 \tau-1)}\left(\frac{2 \tau}{2 \tau-1}\right)^{\tau}=\frac{16 \sqrt{3}}{9}>\frac{1}{2}$,

so the conditions in theorem 2 are satisfied and the characteristic equation is

$$
F(\lambda)=(\lambda-1) \lambda^{-1}\left(\lambda^{2}-\frac{1}{10}\right)+10=0
$$

From the figure 2 we can see the equation (2) has no real root. Therefore all solutions of equation (1) are oscillatory.

Example 3.Consider difference equation $\Delta\left(x(n)-\frac{1}{4} x(n-2)\right)+3 x(n-1)=0$, where $p=\frac{1}{4}, \tau=2$,

$\sigma=1, q=3$. Then $\tau-\sigma=1$ and $q-\tau\left(p^{\frac{1}{\tau}}-1\right)^{2}=\frac{5}{2}>0$

so the conditions in theorem 1 are satisfied and the characteristic equation is

$$
F(\lambda)=(\lambda-1) \lambda^{-1}\left(\lambda^{2}-\frac{1}{4}\right)+3=0
$$

From the figure 3 we can see the equation (2) has no real root. Therefore all solutions of equation (1) are oscillatory.

\section{Conclusion}

In this paper, the conditions for oscillation of difference equation [1] were studied through their characteristic equation and some new oscillatory criteria were given. But these criteria are conditions only of sufficient and not of necessary. The sufficient and necessary conditions of the equation are needed to study further.

\section{References}

He, Xinguang, Luo, Zhiguo, \& Li, hua. (2003). Oscillation of neutrul difference equations with positive and nagative coefficients. Journal of Mathematical Study. 36[4]:388-393.

Li, Qiaoluan, Liu, Zhaoshuang, \& Bai, Jingshan. (2004). Oscillation of First-order Neutral Difference Equation. Journal of Hebei Normal University. 28(6): 569-570.

Li, Yumei, Wang, Youbin, \& Fan, Yehua. (2007). Qscillation of First-order Neutral Difference Equation. Math in 
practice and theory. 27(31), 188-191.

PARHIN, \& TRIPATHY A K. (2003). Oscillation of a class of nonlinear neutral difference equation of higher order. $J$. Math.Anal Appl. 28(4): 756-774.

SABER N E. (1995). .An Introduction to Difference Equation. New York: Spring-verlag.

Zhou, Yinggao, \& Tang, Xianhua. (2002). Oscillation of First-order Nonlinear Delay Difference Equations. Acta mathematical Applicatae sinica. 15(3):132-135

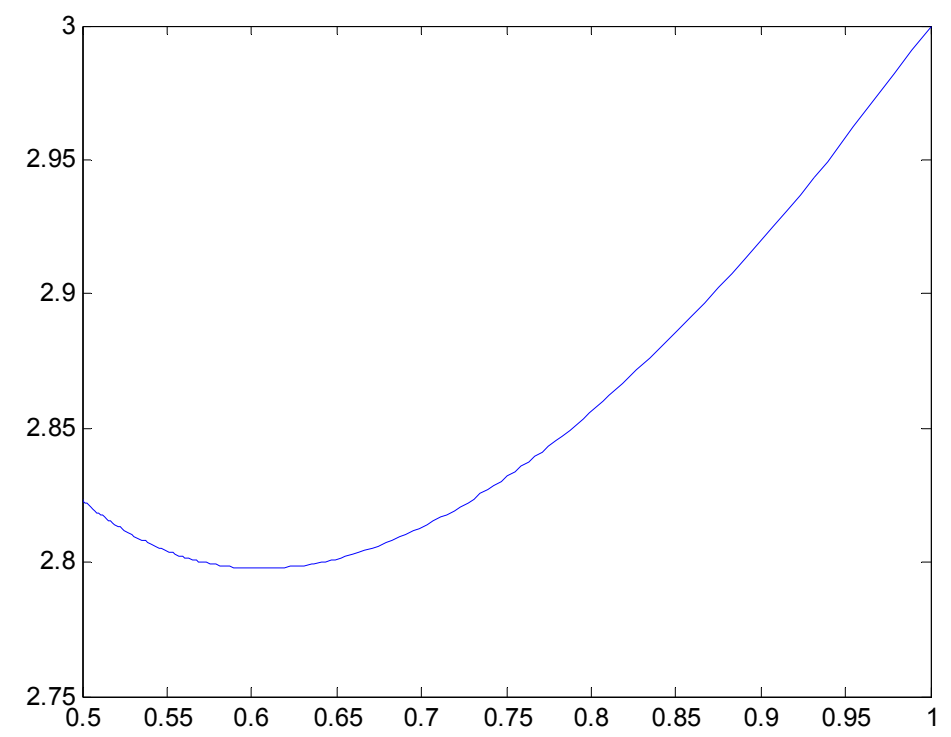

Figure 1. The figure of (3) on interval $\left(\left(\frac{1}{4}\right)^{\frac{1}{2}}, 1\right)$

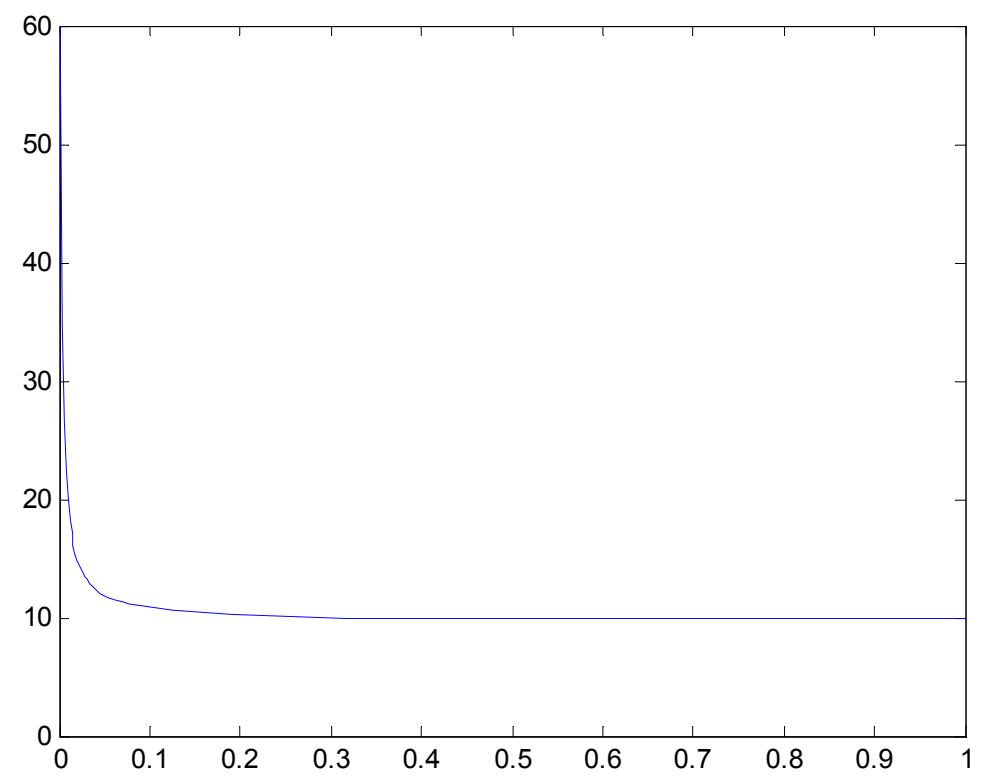

Figure 2. The figure of (4) on interval $(0,1)$ 


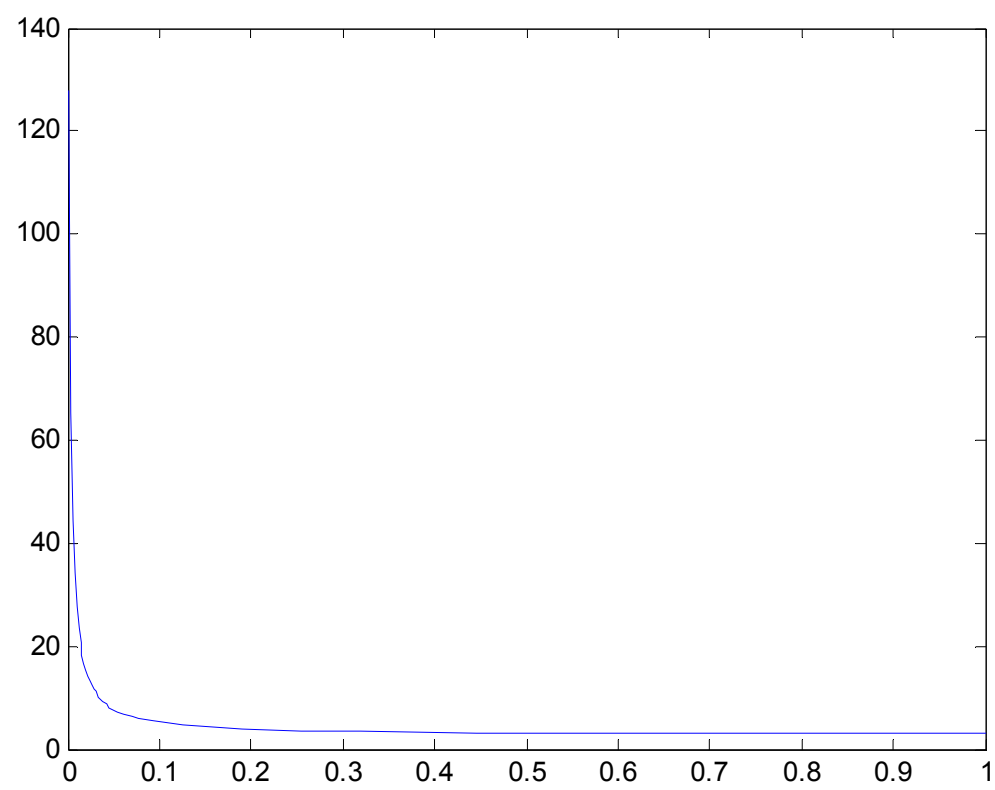

Figure 3. The figure of (5) on interval $(0,1)$ 